\title{
Bluetooth Based Automatic Hotel Service System Using Python
}

\author{
Rajesh Bag, Satyajit Bhowmick, Rahul Ghosh, Abhishek Kumar Gond \\ Department of Electronics and Communication Engineering \\ West Bengal University of Technology (WBUT), Kolkata, India
}

\begin{abstract}
This paper describes a Bluetooth based automatic hotel service system. This paper describes the protocol and provides the implementation details. Nowadays hotels are spending lot of money for the attendance, tracking of the employees and apart from that, there is no fully satisfied service for customers. Instead of using manual power if we apply Bluetooth technology, then the proposed system will cut off the daily wages of hotels. We hope that the implementation and protocol details will aid the wireless technology community in near future.
\end{abstract}

Index: Bluetooth, Python Technology, Arduino.

\section{Introduction}

The project has been modeled by taking Bluetooth scanned data in different times and different places. Using the data, the Bluetooth-Machine interaction is caused. Karen et al. [1] proposed a Bluetooth Security System that acts as a reference to organizations on security capabilities of Bluetooth and demonstrates steps for securing Bluetooth technology effectively. Nateq et al. [2] and Trishna et al. [3] worked on the same basis. Though Bluetooth technology [4] has innumerable benefits, it is also susceptible to denial-of-service attacks, eavesdropping, man-in-the-middle attacks [5], message modification, and resource misappropriation. Python has been used in artificial intelligence [6] tasks. It is a scripting language with module architecture, syntax simplicity and rich text processing tools.

The prioritized objective of the system is to track authorization at department gates which will be controlled by motor actuators and Arduino. It calculates the entry, exit and time duration of an authorized person (employee) within the perimeters of the zone. Data transfer within interlinked departments can be done efficiently to provide total hotel service interface by employee for customer through the Bluetooth device. All the employees and staffs are already given Bluetooth devices by one each for authenticating with the hotel. For the customer to achieve the facility, he/she (customer) has to collect a Bluetooth device from the reception after booking room, then it is connected to the server and the customer will be able to use this system.

The next section describes the hardware setup of our system. Section III presents the protocol of the system. Section IV discusses the experimental evaluation and results. Section V presents the conclusion \& future work of the paper.

\section{Software \& Hardware Description}

The hardware platform of our research consists of PYTHON PROGRAMMING SOFTWARE, BLUETOOTH BATCH, ARDUINO and SERVO MOTOR. In the following sections we will discuss in more details about the individual components.

\section{A. Python}

Python [7] is a high-level, general-purpose programming language utilized in modern day hardware programming whose design emphasizes code readability. Python combines remarkable efficiency with very clear syntax and its standard library is commendably large and comprehensive. Its use of indentation for block delimiters is a unique feature among popular programming languages.

Python essentially supports multifarious programming paradigms primarily but it is not limited to object-oriented, imperative and to a lesser extent, functional programming styles. It features an entirely dynamic type system and automatic memory management, similar to that of Scheme, Ruby, and Perl. Python is often used as a scripting language just like other dynamic languages. Python interpreters are simple and easily available for many operating systems.

\section{B. Bluetooth Batch}

Bluetooth is an open wireless based technology standardized for exchanging data over short geographic distances (using short wavelength radio transmissions in the ISM band ranging between $2400-2480 \mathrm{MHz}$ ) in the ranges of meters. It works equally well with fixed as well as mobile devices, thus creating personal area networks (PANs) that comes with high levels of security, initially created by telecom vendor Ericsson in 1994. 
As a matter of fact, the design was originally conceived as a wireless alternative to RS-232 [8] data cables. It can also interconnect several devices, thus overcoming problems of synchronization.

Bluetooth is actually a standard wire-replacement communication protocol that has been primarily designed for low power consumption, with a short range (power-class-dependent, but effective ranges vary in practice) and built on low-cost transceiver microchips installed within each device.

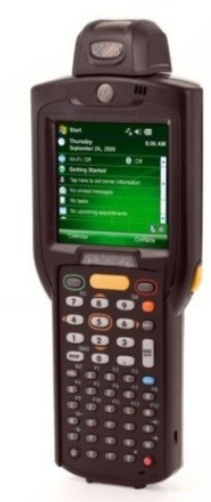

Figure 1: Bluetooth Batch

It is universally supported and used in innumerable modern day products by over 3000 companies, including the likes of Sony Ericsson, Nokia, Intel, IBM, Toshiba, Apple, Microsoft and many more.

The Bluetooth address has a unique feature that comes with a 48-bit device identifier. The first 3 bytes of the address are always assigned to a specific manufacturer by IEEE and the last 3 bytes are randomly allocated by the manufacturer [9].

\section{Arduino}

Arduino [10] is essentially an open-source electronics prototyping platform that utilizes flexible and easy-to-use hardware and software thus creating interactive objects or environments. Artists, designers and technical hobbyists have undoubtedly a special liking for it.

An Arduino is integrated to sense the environment by receiving inputs from variety of sensors and can affect its surroundings by controlling actuators. The microcontroller is programmed using the Arduino programming language (based on Wiring) and the Arduino development environment (based on Processing).

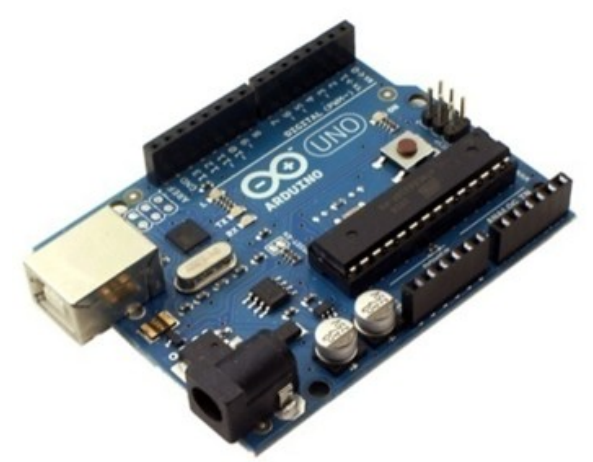

Figure 2: Arduino

Arduino projects are either stand-alone or communicable with software running on a computer (e.g. Flash, Processing, and Max MSP).The boards can be built or purchased preassembled and the software can be downloaded from the internet for free. The hardware reference designs (CAD files [11]) are available under an open-source license, which are free to be adapted.

The Arduino board is based on the integration of the microcontroller's I/O pins which are used by other circuits. The board provides 12 digital $\mathrm{I} / \mathrm{O}$ pins, six pins of these are for producing pulse-width modulated signals and other six are for analog inputs. These pins are situated on the top of the board via female headers whose sizes are about 0.10 -inch $(2.5 \mathrm{~mm})$.

Arduino received an Honorary Mention that is in the Digital Communities section of the 2006 Ars Electronica Prix. The Arduino team is comprised of the likes of Massimo Banzi, David Cuartielles, Tom Igoe, Gianluca Martino and David Mellis [12]. These men are credited with the development and creation of the Arduino based concept that has revolutionized today's world of innovative technology and robotics. 


\section{Motor Controlled Door}

Motor actuators add convenience and save time compared to a manually operated door. In order to trigger doors to operate, controllers (ARDUINO) are used for almost any type of gate or door including roll-up door or parking lot security arm to ensure security, reliability and convenience for the users.

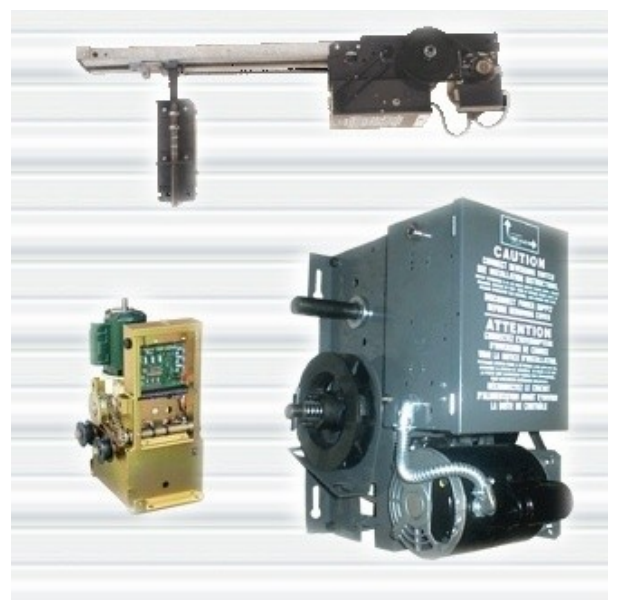

Figure 3: Motor Controlled Door

However, there is a problem in interfacing between Arduino and motor. The Arduino has no ability for controlling more than $40 \mathrm{ma}$ of current. So, an external power source is needed to run the motor. Motor actuators can be a wired combination keypad, wireless fob, computer, smoke and fire sensor, automobile sensor or simply a push button. At CDC we have experienced all these systems to conveniently and reliably open and close doors or gates [13]. CDC services support most brands of motor operators for particular application.

The motor's motion is controlled in several ways. Pulse-width modulation (PWM) is the best of them. It can be applied in Arduino in various ways. Using PWM registers, the duty cycle and frequency can be produced. It minimizes the other controllers' complications. The PWM signal is a digital square wave in where the duty cycle can be changed from 0 to $100 \%$. Here the frequency is to be constant. However, this technique has some drawbacks. First of all, there are some delays or interrupts in PWM operation. It will affect the timing. Secondly due to real time task you can't leave the output while the processor is running on.

(FOR CUSTOMER)

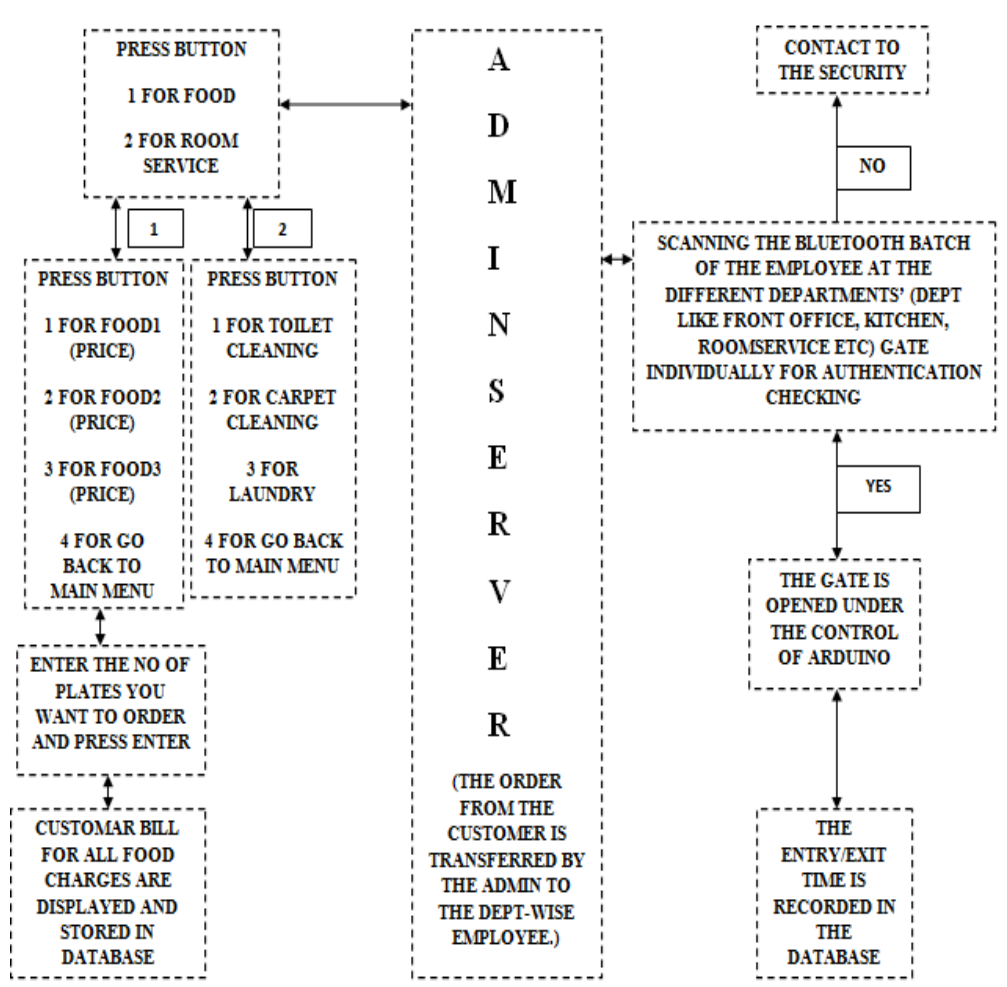

Figure 4: Block Diagram of the System 


\section{Protocol}

The purpose of the total system is servicing in any large hotels, tracking employees and communicating with the customers and also recording every employee's and customer's details in a database. Every employee and customer will be provided one Bluetooth device with display system. Every device has its own MAC ID [14] by which everyone can be serviced individually.

After booking room, if the customer wants to order food, he/she can check the food items available in the hotel from the food details part of the system and price per plate of the items which are already mentioned. Now if the customer requires room service, he/she can get the requirement from the room service function provided in the system.

In any large hotel there are thousands of employees and various departments (dept. like front office, kitchen, room service etc.). When an employee wants to enter in a particular department, the installed scanner scans the employee by his or her Bluetooth device and then takes its MAC ID. If the person belongs to that department and if the MAC ID is found and matches with the database of the hotel, the gate will be opened automatically under the control of Arduino and servo motor. If the MAC ID does not exist in the hotel's database or no Bluetooth device is found, it displays that the employee is unauthorized and should contact to the security. For any purpose if any employee wants to go from one department to another, he/she has to take permission from the admin. Then the admin just adds the MAC ID of that particular employee for the desired department temporarily. Then the employee can go to another department. If the admin wants to give any instruction to any particular person, it can send message through the Bluetooth device, as it has a display. So loss of messages will be reduced

In the whole area of the hotel there are so many scanners to scan all the devices in every moment to track every person. The whole system is automatically handled to serve every customer in every moment and how many days the customer spend will also be recorded in the database which is arranged by the system itself with date and employees' time, date, departments will also be recorded.

\section{Experimental Evaluation}

The testing of the employees MAC ID at the department gate gives us various plots with the no. of inputs (i.e., no. of MAC ID'S) as x-axis and time (in sec) as y-axis. In the Figure 5(a), the line is constantly increasing with respect to its axis i.e., no. of inputs is directly proportional with time axis and in the Figure 5(b), the line is proportionally increasing up to some extent but a sudden fall comes due to the locality of reference i.e., changing the no. of inputs keeping fixed the ranges. After that the line is proportionally increasing.

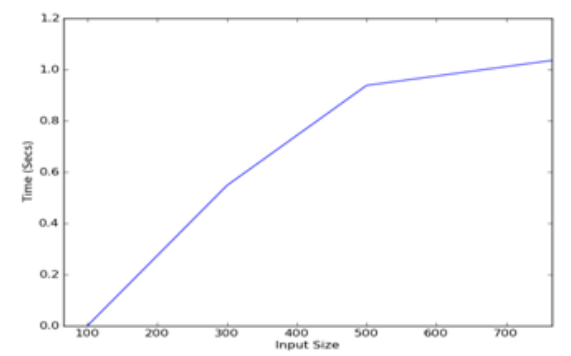

Figure 5(a): No. of MAC Inputs vs. Time Graph

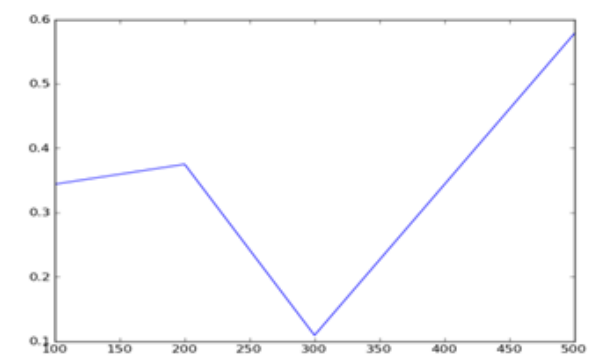

Figure 5(b): Graphical pattern with changing number of MAC inputs

\section{Conclusion \& Future Work}

The system was tested in lab environment and it works as per expectation. The experimental results provided in the report show the performance of this system. This system has an ability to serve every customer by food supplying, room servicing and also detect an authorized person having Bluetooth batches at different 
departments. This system records entry time, exit time and duration spent in a specific department by an authorized person. The main advantages of this system are low installation cost and low maintenance cost. We hope that this system will help the wireless technology community in near future.

The designed system lacks some security features. As the system stores hotel service and employee information, the system needs to be secure enough from outside attackers. We can import the security features within the system in order to make it more secure and to prevent the vulnerability. We can enhance its' performance by using more sophisticated data structure and search algorithm.

\section{Acknowledgement}

The authors would also like to extend their whole-hearted gratitude towards Mr. Avranil Tah of ESL, Salt Lake, West Bengal, India for his extensive guidance and intellectual support provided during the execution of the research work.

\section{References}

[1] KAREN SCARFONE \& JOHN PADGETTE, "Guide to Bluetooth Security", U.S. Department of Commerce, National Institute of Standards and Technology (NIST), Special Publication 800-121, September2008,[online],available: http://bluetoothpentest.narod.ru/doc/guide to bluetooth security.pdf

[2] Nateq Be-Nazir Ibn Minar \& Mohammed Tarique, "BLUETOOTH SECURITY THREATS AND SOLUTIONS: A SURVEY", International Journal of Distributed and Parallel Systems (IJDPS) Vol.3, No.1, January 2012, pages127-148.

[3] Trishna Panse, Vivek Kapoor, "A REVIEW ON SECURITY MECHANISM OF BLUETOOTH COMMUNICATION", Trishna Panse et al, / (IJCSIT) International Journal of Computer Science and Information Technologies, Vol. 3 (2), 2012 , pages 3419-3422.

[4] Bluetooth Technology, [online], Available: http://www.bluetooth.com/Pages/what-is-bluetooth-technology.aspx

[5] "Man in the Middle" Attacks on Bluetooth, [online], Available: http://link.springer.com/chapter/10.1007\%2F978-3-54045126-6 11\#page-1

[6] Artificial Intelligence, [online], Available; http://artint.info/html/ArtInt.html

[7] Python, [online], Available: http://docs.python.org/2/tutorial/

[8] RS-232, [online], Available: http://en.wikipedia.org/wiki/RS-232

[9] Bluetooth security review, [online], Available: http://www.symantec.com/connect/articles/bluetooth-security-review-part-1

[10] Arduino, [online], Available: http://www.arduino.cc/

[11] CAD Tool, [online], Available: http://en.wikipedia.org/wiki/Computer-aided design

[12] Ars Electronica Archive, [online], Available:

http://90.146.8.18/en/archives/picture ausgabe 02 new.asp?iAreaID=298\&showAreaID=318

[13] Inductive load control, [online], Available: http://workshops.ist.utl.pt/arduino-lessons/lesson-ii-3-inductive-load-control/

[14] MAC Address, [online], Available: http://www.webopedia.com/TERM/M/MAC_address.html 